“@ 2018 IEEE. Personal use of this material is permitted. Permission from IEEE must be obtained for all other uses, in any current or future media, including reprinting/republishing this material for advertising or promotional purposes, creating new collective works, for resale or redistribution to servers or lists, or reuse of any copyrighted component of this work in other works." 


\title{
Separation between quantum Lovász number and entanglement-assisted zero-error classical capacity
}

\author{
Xin Wang ${ }^{1 *}$ and Runyao Duan ${ }^{1,2 \dagger}$ \\ ${ }^{1}$ Centre for Quantum Software and Information, School of Software, \\ Faculty of Engineering and Information Technology, \\ University of Technology Sydney, NSW 2007, Australia and \\ ${ }^{2}$ UTS-AMSS Joint Research Laboratory for Quantum Computation and Quantum Information Processing, \\ Academy of Mathematics and Systems Science, \\ Chinese Academy of Sciences, Beijing 100190, China ${ }^{\ddagger}$
}

\begin{abstract}
Quantum Lovász number is a quantum generalization of the Lovász number in graph theory. It is the best known efficiently computable upper bound of the entanglementassisted zero-error classical capacity of a quantum channel. However, it remains an intriguing open problem whether quantum entanglement can always enhance the zero-error capacity to achieve the quantum Lovász number. In this paper, by constructing a particular class of qutrit-to-qutrit channels, we show that there exists a strict gap between the entanglement-assisted zero-error capacity and the quantum Lovász number. Interestingly, for this class of quantum channels, the quantum generalization of fractional packing number is strictly larger than the zero-error capacity assisted with feedback or no-signalling correlations, which differs from the case of classical channels.
\end{abstract}

\section{INTRODUCTION}

A fundamental problem of information theory is to determine the capability of a communication channel to deliver messages from the sender to the receiver. While the conventional information theory focuses on sending messages with asymptotically vanishing errors [1], Shannon also investigated this problem in the zero-error setting and described the zero-error capacity of a channel as the maximum rate at which it can be used to transmit information with a zero probability of error [2]. Zero-error information theory [2,3] concerns the asymptotic combinatorial problems, most of which are difficult and unsolved.

Recently the zero-error information theory has been studied in the quantum setting and many new phenomena were observed. One remarkable result is the super-activation of the zero-error classical/quantum capacities of quantum channels [4-8]. Another important result is that, for some classical channels, quantum entanglement can be used to improve the zero-error capacity $[9,10]$, while there is no such advantage for the normal capacity [1]. Furthermore, there are more kinds of capacities when considering auxiliary resources, such as the shared entanglement [4, 9-14], the no-signalling correlations [15-22], and the feedback assistance [2, 23]. All of these capacities are only partially understood, and the zero-error information theory of quantum channels seems more complex than that of classical channels.

To study the zero-error communication via quantum channels, the so-called "noncommutative graph theory" was introduced in [11]. The non-commutative graph (an object based on an operator system) associated with a quantum channel fully captures the zero-error communication properties of this channel [11], thus playing a similar role to confusability graph in the

\footnotetext{
${ }^{*}$ Electronic address: xin.wang-8@student.uts.edu.au

${ }^{\dagger}$ Electronic address: runyao.duan@uts.edu.au

${ }^{\ddagger}$ A preliminary version of this paper was presented as a contributed talk at the 16th Asian Quantum Information Science Conference (AQIS'16).
} 
classical case. It is well-known that the zero-error capacity is extremely difficult to compute for both classical and quantum channels [24]. Nevertheless, the zero-error capacity of a classical channel is upper bounded by the Lovász number [25] while the zero-error capacity of a quantum channel is upper bounded by the quantum Lovász number [11]. Furthermore, the entanglementassisted zero-error capacity of a classical channel is also upper-bounded by the Lovász number [11,27], and this result can be generalized to quantum channels by using the quantum Lovász number [11].

A more general problem is the simulation of a channel, which concerns how to use a channel $\mathcal{N}$ from Alice $(A)$ to Bob $(B)$ to simulate another channel $\mathcal{M}$ also from $A$ to $B$ [28]. Shannon's noisy channel coding theorem determines the capability of any noisy channel $\mathcal{N}$ to simulate a noiseless channel [1] and the reverse Shannon theorem was proved in [29]. The quantum reverse Shannon theorem was proved recently $[30,31]$, which states that any quantum channel can be simulated by an amount of classical communication equal to its entanglement-assisted capacity assisted with free entanglement. In the zero-error setting, there is a kind of reversibility between the zero-error capacity and simulation cost in the presence of no-signalling correlations [16]. More recently, the no-signalling-assisted (NS-assisted) zero-error simulation cost of a quantum channel was introduced in [17].

An intriguing open problem in zero-error information theory is whether the entanglementassisted zero-error capacity always coincides with the quantum Lovász number for a classical or quantum channel, which is frequently mentioned in [10,11, 16, 27, 32, 33]. If they are equal, it will imply that the entanglement-assisted zero-error capacity is additive, while the unassisted case is not [34].

In this paper, we resolve the above open problem for quantum channels. To be specific, we construct a class of qutrit-to-qutrit channels for which the quantum Lovász number is strictly larger than the entanglement-assisted zero-error capacity. We utilize the one-shot NS-assisted zero-error capacity and simulation cost to determine the asymptotic NS-assisted zero-error capacity in this case, which is potentially larger than the entanglement-assisted zero-error capacity. An interesting fact is that this class of channels are reversible in a strong sense. To be specific, for this class of channels, the one-shot NS-assisted zero-error capacity and simulation cost are identical. We then give a closed formula for the quantum Lovász number for this class of channels, and use it to conclude that there is a strict gap between the quantum Lovász number and the entanglementassisted zero-error capacity. For this class of channels, we also find that the quantum fractional packing number is strictly larger than the feedback-assisted or NS-assisted zero-error capacity, while these three quantities are equal to each other for any classical channel [16].

\section{PRELIMINARIES}

In the following, we will frequently use symbols such as $A$ (or $A^{\prime}$ ) and $B$ (or $B^{\prime}$ ) to denote the (finite-dimensional) Hilbert spaces associated with Alice and Bob, respectively. The set of linear operators over $A$ is denoted by $\mathcal{L}(A)$. A quantum channel $\mathcal{N}$ from $A$ to $B$ is simply a completely positive and trace-preserving $(\mathrm{CPTP})$ linear map from $\mathcal{L}(A)$ to $\mathcal{L}(B)$, with a Choi-Kraus operator sum representation $\mathcal{N}(\rho)=\sum_{k} E_{k} \rho E_{k}^{\dagger}$. where $\sum_{k} E_{k}^{\dagger} E_{k}=\mathbb{1}_{A}$. The Choi-Kraus operator space of $\mathcal{N}$ is denoted by

$$
K=K(\mathcal{N}):=\operatorname{span}\left\{E_{k}\right\}
$$

Such space is alternatively called "non-commutative bipartite graph" since it determines the zeroerror capacity of a quantum channel in the presence of noiseless feedback [23], which plays a similar role to the bipartite graph of a classical channel. The Choi-Jamiołkowski matrix of $\mathcal{N}$ : 
$\mathcal{L}\left(A^{\prime}\right) \rightarrow \mathcal{L}(B)$ is $J_{A B}=\sum_{i j}|i\rangle\left\langle\left. j\right|_{A} \otimes \mathcal{N}\left(|i\rangle\left\langle\left. j\right|_{A^{\prime}}\right)=\left(\mathrm{id}_{A} \otimes \mathcal{N}\right)|\Phi\rangle\langle\Phi|\right.\right.$, where $A$ and $A^{\prime}$ are isomorphic Hilbert spaces with respective orthonormal basis $\left\{|i\rangle_{A}\right\}$ and $\left\{|j\rangle_{A^{\prime}}\right\},|\Phi\rangle=\sum_{i}|i\rangle_{A}|i\rangle_{A^{\prime}}$ and id $\operatorname{id}_{A}$ is the identity map. We denote $P_{A B}$ as the projection onto the support of $J_{A B}$, which is the subspace $(\mathbb{1} \otimes K)|\Phi\rangle$. The non-commutative graph [11] of $\mathcal{N}$ is defined by the operator subspace

$$
S:=K^{\dagger} K=\operatorname{span}\left\{E_{j}^{\dagger} E_{k}: j, k\right\}<\mathcal{L}\left(A^{\prime}\right),
$$

where $S<\mathcal{L}\left(A^{\prime}\right)$ means that $S$ is a subspace of $\mathcal{L}\left(A^{\prime}\right)$.

The one-shot zero-error capacity of a quantum channel $\mathcal{N}$ is the maximum number of inputs such that the receiver can perfectly distinguish the corresponding output states. The output states can be perfectly distinguished if and only if they are orthogonal. This one-shot zero-error capacity can be equivalently defined as the independence number $\alpha(S)$ of the non-commutative graph [11] of $\mathcal{N}$, i.e., the maximum size of a set of orthogonal unit vectors $\left\{\left|\phi_{m}\right\rangle: m=1, \ldots, M\right\}$ such that

$$
\forall m \neq m^{\prime},\left|\phi_{m}\right\rangle\left\langle\phi_{m}^{\prime}\right| \in S^{\perp} .
$$

The zero-error capacity is given by regularization of $\alpha(S)$, i.e.,

$$
C_{0}(\mathcal{N})=C_{0}(S)=\sup _{n \rightarrow \infty} \frac{1}{n} \log \alpha\left(S^{\otimes n}\right) .
$$

Throughout this paper, log denotes the binary logarithm $\log _{2}$. The sup in Eq. (1) can be replaced by lim based on the lemma about existence of limits in [35].

The entanglement-assisted independence number $\widetilde{\alpha}(S)$ [11] is motivated by the scenario where sender and receiver share entangled state beforehand and it quantifies the maximum number of distinguishable messages that can be sent via the channel $\mathcal{N}$ with graph $S$ when shared entanglement is free. To be specific, $\widetilde{\alpha}(S)$ is the maximum integer $M$ such that there exist Hilbert spaces $A_{0}, B_{0}$ and a state $\sigma \in \mathcal{L}\left(A_{0} \otimes B_{0}\right)$, and CPTP maps $\mathcal{E}_{m}: \mathcal{L}\left(A_{0}\right) \rightarrow \mathcal{L}(A)(m=1, \ldots, N)$ such that the $N$ output states $\rho_{m}=\left(\mathcal{N} \circ \mathcal{E}_{m} \otimes \operatorname{id}_{B_{0}}\right) \sigma$ are orthogonal. The entanglement-assisted zero-error capacity of $S$ is given by regularization of $\widetilde{\alpha}(S)$, i.e.,

$$
C_{0 \mathrm{E}}(\mathcal{N})=C_{0 \mathrm{E}}(S)=\sup _{n \rightarrow \infty} \frac{1}{n} \log \widetilde{\alpha}\left(S^{\otimes n}\right) .
$$

For any non-commutative graph $S<\mathcal{L}(A)$, the quantum Lovász number $\widetilde{\vartheta}(S)$ was introduced as a quantum analog of the Lovász number in [11]. It can be formalized by semidefinite programming (SDP) [11] as follows:

$$
\begin{aligned}
\widetilde{\vartheta}(S)= & \max \langle\Phi|(\mathbb{1} \otimes \rho+T)| \Phi\rangle \\
\text { s.t. } & T \in S^{\perp} \otimes \mathcal{L}\left(A^{\prime}\right), \quad \operatorname{Tr} \rho=1, \\
& \mathbb{1} \otimes \rho+T \geq 0, \quad \rho \geq 0,
\end{aligned}
$$

where $|\Phi\rangle=\sum_{i}|i\rangle_{A}|i\rangle_{A^{\prime}}$. Note that SDP can be solved by polynomial-time algorithms [26, 36] in usual and it has many other applications in quantum information theory (e.g., [37-44]). More details about SDP can be found in [45]. The dual SDP of $\widetilde{\vartheta}(S)$ is given by

$$
\begin{aligned}
& \widetilde{\vartheta}(S)= \min \left\|\operatorname{Tr}_{A} Y\right\|_{\infty} \\
& \text { s.t. } Y \in S \otimes \mathcal{L}\left(A^{\prime}\right), \quad Y \geq|\Phi\rangle\langle\Phi| .
\end{aligned}
$$

The operator norm $\|R\|_{\infty}$ is defined as the maximum eigenvalue of $\sqrt{R^{+} R}$. By strong duality, the optimal values of the primal and dual SDPs of $\widetilde{\vartheta}(S)$ coincide. Furthermore, $\widetilde{\vartheta}(S)$ was proved to be an upper bound of $C_{0 \mathrm{E}}(S)$ [11],

$$
C_{0}(S) \leq C_{0 \mathrm{E}}(S) \leq \log \widetilde{\vartheta}(S) .
$$


Moreover, for a quantum channel $\mathcal{N}$ with non-commutative graph $S$, the quantum Lovász number of $\mathcal{N}$ is naturally given by the quantum Lovász number of $S$,

$$
\widetilde{\vartheta}(\mathcal{N})=\widetilde{\vartheta}(S) .
$$

The no-signalling correlations arises in the research of the relativistic causality of quantum operations [46-49] and Cubitt et al. [16] first introduced classical no-signalling correlations into the zero-error communication via classical channels and proved that the fractional packing number of the bipartite graph induced by the channel equals to the zero-error capacity of the channel. Recently, quantum no-signalling correlations were introduced into the zero-error communication via quantum channels in [17] and the one-shot NS-assisted zero-error classical capability (quantified as the number of messages) was formulated as the following SDP:

$$
\begin{aligned}
& \Upsilon(\mathcal{N})=\Upsilon(K)=\max \operatorname{Tr} R_{A} \\
& \text { s.t. } 0 \leq U_{A B} \leq R_{A} \otimes \mathbb{1}_{B}, \\
& \operatorname{Tr}_{A} U_{A B}=\mathbb{1}_{B}, \\
& \operatorname{Tr} P_{A B}\left(R_{A} \otimes \mathbb{1}_{B}-U_{A B}\right)=0,
\end{aligned}
$$

where $P_{A B}$ denotes the projection onto $(\mathbb{1} \otimes K)|\Phi\rangle$. The asymptotic NS-assisted zero-error capacity is given by the regularization:

$$
C_{0, \mathrm{NS}}(\mathcal{N})=C_{0, \mathrm{NS}}(K)=\sup _{n \rightarrow \infty} \frac{1}{n} \log \Upsilon\left(K^{\otimes n}\right) .
$$

A remarkable feature of NS-assisted zero-error capacity is that one bit noiseless communication can fully activate any classical-quantum channel to achieve its asymptotic capacity [50].

The zero-error simulation cost of a quantum channel in the presence of quantum no-signalling correlations was introduced in [17] and formalized as SDPs. To be specific, for the quantum channel $\mathcal{N}$ with Choi-Jamiołkowski matrix $J_{A B}$, the NS-assisted zero-error simulation cost of $\mathcal{N}$ is given by

$$
S_{0, \mathrm{NS}}(\mathcal{N})=-H_{\min }(A \mid B)_{J_{A B}}:=\log \Sigma(\mathcal{N}),
$$

where

$$
\begin{aligned}
\Sigma(\mathcal{N})= & \min \operatorname{Tr} T_{B}, \\
& \text { s.t. } J_{A B} \leq \mathbb{1}_{A} \otimes T_{B},
\end{aligned}
$$

and $H_{\min }(A \mid B)_{J_{A B}}$ is the so-called conditional min-entropy [51,52]. By the fact that the conditional min-entropy is additive [51], the asymptotic NS-assisted zero-error simulation cost is given by

$$
S_{0, \mathrm{NS}}(\mathcal{N})=\log \Sigma(\mathcal{N}) .
$$

Furthermore, noting that the NS assistance is stronger than the entanglement assistance, the capacities and simulation cost of a quantum channel introduced above obey the following inequality:

$$
C_{0} \leq C_{0 \mathrm{E}} \leq C_{0, \mathrm{NS}} \leq C_{\mathrm{E}} \leq S_{0, \mathrm{NS}},
$$

where $C_{\mathrm{E}}$ is the entanglement-assisted classical capacity [29]. 


\section{GAP BETWEEN QUANTUM LOVÁSZ NUMBER AND ENTANGLEMENT-ASSISTED ZERO-ERROR CAPACITY}

In this section, we are going to show the gap between the quantum Lovász number and the entanglement-assisted zero-error capacity. The difficulty in comparing $C_{0 \mathrm{E}}$ and the quantum Lovász number is that there are few channels whose entanglement-assisted zero-error capacity is known. In fact, $C_{0 \mathrm{E}}$ is even not known to be computable. The problem whether there exists a gap between them was a prominent open problem in the area of zero-error quantum information theory.

Our approach to the above problem is to construct a particular class of channels and considering the NS-assisted zero-error capacity, which is potentially larger than the entanglement-assisted case. To be specific, the class of channels we use is $\mathcal{N}_{\alpha}(\rho)=E_{\alpha} \rho E_{\alpha}^{\dagger}+D_{\alpha} \rho D_{\alpha}^{\dagger}(0<\alpha \leq \pi / 4)$ with

$$
\begin{aligned}
E_{\alpha} & =\sin \alpha|0\rangle\langle 1|+| 1\rangle\langle 2|, \\
D_{\alpha} & =\cos \alpha|2\rangle\langle 1|+| 1\rangle\langle 0| .
\end{aligned}
$$

This qutrit-qutrit channel $\mathcal{N}_{\alpha}$ is motivated in the similar sipirt of the amplitutde damping channel, which exhibits a significant differnece from the classical-quantum channels.

The first Choi-Kraus operator $E_{\alpha}$ annihilates the ground state $|0\rangle\langle 0|$ :

$$
E_{\alpha}|0\rangle\langle 0| E_{\alpha}^{\dagger}=0
$$

and it decays the state $|1\rangle\langle 1|$ to the ground state $|0\rangle\langle 0|$ :

$$
E_{\alpha}|1\rangle\left\langle 1\left|E_{\alpha}^{\dagger}=\sin ^{2} \alpha\right| 0\right\rangle\langle 0| .
$$

Meanwhile, $E_{\alpha}$ also transfer the state $|2\rangle\langle 2|$ to $|1\rangle\langle 1|$, i.e., $E_{\alpha}|2\rangle\left\langle 2\left|E_{\alpha}^{\dagger}=\right| 1\right\rangle\langle 1|$. On the other hand, the choice of $D_{\alpha}$ above ensures that

$$
E_{\alpha}^{\dagger} E_{\alpha}+D_{\alpha}^{\dagger} D_{\alpha}=\mathbb{1}
$$

which means that the operators $E_{\alpha}$ and $D_{\alpha}$ are valid Kraus operators for a quantum channel.

The Choi-Jamiołkowski matrix of $\mathcal{N}_{\alpha}$ is given by

$$
J_{\alpha}=\left(1+\sin ^{2} \alpha\right)\left|u_{\alpha}\right\rangle\left\langle u_{\alpha}\left|+\left(1+\cos ^{2} \alpha\right)\right| v_{\alpha}\right\rangle\left\langle v_{\alpha}\right|,
$$

where

$$
\begin{aligned}
& \left|u_{\alpha}\right\rangle=\frac{\sin \alpha}{\sqrt{1+\sin ^{2} \alpha}}|10\rangle+\frac{1}{\sqrt{1+\sin ^{2} \alpha}}|21\rangle, \\
& \left|v_{\alpha}\right\rangle=\frac{\cos \alpha}{\sqrt{1+\cos ^{2} \alpha}}|12\rangle+\frac{1}{\sqrt{1+\cos ^{2} \alpha}}|01\rangle .
\end{aligned}
$$

Then, the projection onto the support of $J_{\alpha}$ is

$$
P_{\alpha}=\left|u_{\alpha}\right\rangle\left\langle u_{\alpha}|+| v_{\alpha}\right\rangle\left\langle v_{\alpha}\right|
$$

We first prove that both NS-assisted zero-error capacity and simulation cost of $\mathcal{N}_{\alpha}$ are exactly two bits.

Proposition 1 For the channel $\mathcal{N}_{\alpha}(0<\alpha \leq \pi / 4)$,

$$
C_{0, \mathrm{NS}}\left(\mathcal{N}_{\alpha}\right)=C_{\mathrm{E}}\left(\mathcal{N}_{\alpha}\right)=S_{0, \mathrm{NS}}\left(\mathcal{N}_{\alpha}\right)=2 .
$$


Proof First, we show that Alice can trasmit at least 2 bits prefectly to Bob with a single use of $\mathcal{N}_{\alpha}$ and the NS-assistance. The approach is to construct a feasible solution of the SDP (6) of the one-shot NS-assisted zero-error capacity. To be specific, suppose that $R_{A}=2\left(\cos ^{2} \alpha|0\rangle\langle 0|+| 1\rangle\langle 1|+\right.$ $\left.\sin ^{2} \alpha|2\rangle\langle 2|\right)$ and

$$
\begin{aligned}
U_{A B}= & \cos ^{2} \alpha|01\rangle\left\langle 01\left|+\sin ^{2} \alpha\right| 21\right\rangle\langle 21|+| 10\rangle\langle 10|+| 12\rangle\langle 12| \\
& +\sin \alpha(|10\rangle\langle 21|+| 21\rangle\langle 10|)+\cos \alpha(|01\rangle\langle 12|+| 12\rangle\langle 01|) .
\end{aligned}
$$

One can simply check that $R_{A} \otimes \mathbb{1}_{B}-U_{A B} \geq 0, \operatorname{Tr}_{A} U_{A B}=\mathbb{1}_{B}$ and $P_{\alpha}\left(R_{A} \otimes \mathbb{1}_{B}-U_{A B}\right)=0$. Therefore, $\left\{R_{A}, U_{A B}\right\}$ is a feasible solution to $\operatorname{SDP}(6)$ of $\Upsilon\left(\mathcal{N}_{\alpha}\right)$, which means that

$$
C_{0, \mathrm{NS}}\left(\mathcal{N}_{\alpha}\right) \geq \log \Upsilon\left(\mathcal{N}_{\alpha}\right) \geq \log \operatorname{Tr} R_{A}=2 .
$$

Second, we prove that the one-shot NS-assisted simulation cost of $\mathcal{N}_{\alpha}$ is at amost 2 bits. We utilize the SDP (9) of one-shot NS-assisted simulation cost and choose

$$
T_{B}=2\left(\sin ^{2} \alpha|0\rangle\langle 0|+| 1\rangle\left\langle 1\left|+\cos ^{2} \alpha\right| 2\right\rangle\langle 2|\right) .
$$

It can be checked that $\mathbb{1} \otimes T_{B}-J_{\alpha} \geq 0$. Thus, $T_{B}$ is a feasible solution to SDP (9) of $\Sigma\left(\mathcal{N}_{\alpha}\right)$, which means that

$$
S_{0, \mathrm{NS}}\left(\mathcal{N}_{\alpha}\right) \leq \log \Sigma\left(\mathcal{N}_{\alpha}\right) \leq \log \operatorname{Tr} T_{B}=2 .
$$

Finally, combining Eq. (16), Eq. (18) and Eq. (11), it is clear that

$$
C_{0, \mathrm{NS}}\left(\mathcal{N}_{\alpha}\right)=C_{\mathrm{E}}\left(\mathcal{N}_{\alpha}\right)=S_{0, \mathrm{NS}}\left(\mathcal{N}_{\alpha}\right)=2 .
$$

We then solve the exact value of the quantum Lovász number of $\mathcal{N}_{\alpha}$.

Proposition 2 For the channel $\mathcal{N}_{\alpha}(0<\alpha \leq \pi / 4)$,

$$
\widetilde{\vartheta}\left(\mathcal{N}_{\alpha}\right)=2+\cos ^{2} \alpha+\cos ^{-2} \alpha>4 \text {. }
$$

Proof We first construct a quantum state $\rho$ and an operator $T \in S^{\perp} \otimes \mathcal{L}\left(A^{\prime}\right)$ such that $\mathbb{1} \otimes \rho+T$ is positive semidefinite. Then, we use the primal SDP (3) of $\widetilde{\vartheta}\left(\mathcal{N}_{\alpha}\right)$ to obtain the lower bound of $\widetilde{\vartheta}\left(\mathcal{N}_{\alpha}\right)$.

To be specific, the non-commutative graph of $\mathcal{N}_{\alpha}$ is $S=\operatorname{span}\left\{F_{1}, F_{2}, F_{3}, F_{4}\right\}$ with

$$
\begin{aligned}
& F_{1}=|0\rangle\left\langle 0\left|+\cos ^{2} \alpha\right| 1\right\rangle\langle 1|, \\
& F_{2}=\sin ^{2} \alpha|1\rangle\langle 1|+| 2\rangle\langle 2|, \\
& F_{3}=|0\rangle\langle 2| \text { and } F_{4}=|2\rangle\langle 0| .
\end{aligned}
$$

Let us choose

$$
\rho=\frac{\cos ^{2} \alpha}{1+\cos ^{2} \alpha}|0\rangle\left\langle 0\left|+\frac{1}{1+\cos ^{2} \alpha}\right| 1\right\rangle\langle 1|
$$

and $T=T_{1} \otimes T_{2}+R$, where

$$
\begin{aligned}
T_{1} & =\frac{1}{1+\cos ^{2} \alpha}\left(|0\rangle\left\langle 0\left|-\frac{1}{\cos ^{2} \alpha}\right| 1\right\rangle\left\langle 1\left|+\frac{\sin ^{2} \alpha}{\cos ^{2} \alpha}\right| 2\right\rangle\langle 2|\right), \\
T_{2} & =\cos ^{4} \alpha|0\rangle\langle 0|-| 1\rangle\langle 1|, \\
R & =|00\rangle\langle 11|+| 11\rangle\langle 00| .
\end{aligned}
$$


It is clear that $\rho \geq 0$ and $\operatorname{Tr} \rho=1$. Also, it is easy to see that for any matrix $M \in \mathcal{L}\left(A^{\prime}\right)$ and $j=1,2,3,4$,

$$
\operatorname{Tr} R\left(F_{j} \otimes M\right)=0 .
$$

Meanwhile, noticing that $\operatorname{Tr}\left(T_{1} F_{j}\right)=0$ for $j=1,2,3,4$, we have

$$
T=T_{1} \otimes T_{2}+R \in S^{\perp} \otimes \mathcal{L}\left(A^{\prime}\right) .
$$

Moreover, it is easy to see that

$$
\begin{aligned}
\mathbb{1} \otimes \rho+T= & \cos ^{2} \alpha|00\rangle\left\langle 00\left|+\frac{1}{\cos ^{2} \alpha}\right| 11\right\rangle\langle 11|+| 00\rangle\langle 11| \\
& +|11\rangle\left\langle 00\left|+\frac{\cos ^{2} \alpha-\cos ^{4} \alpha}{1+\cos ^{2} \alpha}\right| 20\right\rangle\langle 20| \\
& +\frac{2 \cos ^{2}-1}{\left(1+\cos ^{2} \alpha\right) \cos ^{2} \alpha}|21\rangle\langle 21| \geq 0 .
\end{aligned}
$$

Then, $\{\rho, T\}$ is a feasible solution to primal SDP (3) of $\widetilde{\vartheta}\left(\mathcal{N}_{\alpha}\right)$. Hence, we have that

$$
\begin{aligned}
\widetilde{\vartheta}\left(\mathcal{N}_{\alpha}\right) & \geq \operatorname{Tr}[|\Phi\rangle\langle\Phi|(\mathbb{1} \otimes \rho+T)] \\
& =\operatorname{Tr}\left[|\Phi\rangle\langle\Phi|\left(\mathbb{1} \otimes \rho+T_{1} \otimes T_{2}+R\right)\right] \\
& =2+\cos ^{2} \alpha+\cos ^{-2} \alpha
\end{aligned}
$$

On the other hand, we find a feasible solution to the dual SDP (4) of $\widetilde{\vartheta}\left(\mathcal{N}_{\alpha}\right)$. It is easy to see that

$$
S^{\perp}=\operatorname{span}\left\{\mathrm{M}_{1}, \mathrm{M}_{2}, \mathrm{M}_{3}, \mathrm{M}_{4}, \mathrm{M}_{5}\right\}
$$

where $M_{1}=|0\rangle\left\langle 1\left|, M_{2}=\right| 1\right\rangle\left\langle 0\left|, M_{3}=\right| 1\right\rangle\left\langle 2\left|, M_{4}=\right| 2\right\rangle\langle 1|$ and $M_{5}=|0\rangle\left\langle 0\left|-\cos ^{-2} \alpha\right| 1\right\rangle\left\langle 1\left|+\tan ^{2} \alpha\right| 2\right\rangle\langle 2|$. Let us choose

$$
Y=Y_{1} \otimes(|0\rangle\langle 0|+| 1\rangle\langle 1|)+Y_{2} \otimes|2\rangle\langle 2|+\frac{1+\cos ^{2} \alpha}{\cos ^{2} \alpha} Y_{3}
$$

with

$$
\begin{aligned}
Y_{1}= & \left(1+\cos ^{2} \alpha\right) \cos ^{-2} \alpha|0\rangle\left\langle 0\left|+\left(1+\cos ^{2} \alpha\right)\right| 1\right\rangle\langle 1|, \\
Y_{2}= & \left(2-\cos ^{-2} \alpha\right)|0\rangle\left\langle 0\left|+\left(\cos ^{-2} \alpha-\sin ^{2} \alpha\right)\right| 1\right\rangle\langle 1| \\
& +\left(1+\cos ^{2} \alpha\right) \cos ^{-2} \alpha|2\rangle\langle 2|, \\
Y_{3}= & |00\rangle\langle 22|+| 22\rangle\langle 00| .
\end{aligned}
$$

It is easy to see that for any matrix $V \in \mathcal{L}\left(A^{\prime}\right)$ and $j=1,2,3,4,5$, we have that

$$
\operatorname{Tr} Y_{3}\left(M_{j} \otimes V\right)=0 .
$$

Meanwhile, since $\operatorname{Tr}\left(Y_{k} M_{j}\right)=0$ for $k=1,2$ and $j=1,2,3,4,5$, we have that

$$
\begin{aligned}
Y & =Y_{1} \otimes(|0\rangle\langle 0|+| 1\rangle\langle 1|)+Y_{2} \otimes|2\rangle\langle 2|+\frac{1+\cos ^{2} \alpha}{\cos ^{2} \alpha} Y_{3} \\
& \in S \otimes \mathcal{L}\left(A^{\prime}\right) .
\end{aligned}
$$


It is also easy to check that $Y-|\Phi\rangle\langle\Phi| \geq 0$. Thus, $Y$ is a feasible solution to SDP (4) of $\widetilde{\vartheta}\left(\mathcal{N}_{\alpha}\right)$. Furthermore, one can simply calculate that

$$
\operatorname{Tr}_{A} Y=\left(2+\cos ^{2} \alpha+\cos ^{-2} \alpha\right) \mathbb{1}_{B}
$$

Therefore,

$$
\widetilde{\vartheta}\left(\mathcal{N}_{\alpha}\right) \leq\left\|\operatorname{Tr}_{A} Y\right\|_{\infty}=2+\cos ^{2} \alpha+\cos ^{-2} \alpha .
$$

Finally, combining Eq. (32) and Eq. (41), we can conclude that

$$
\widetilde{\vartheta}\left(\mathcal{N}_{\alpha}\right)=2+\cos ^{2} \alpha+\cos ^{-2} \alpha \text {. }
$$

Now we are able to show a separation between $\log \widetilde{\vartheta}\left(\mathcal{N}_{\alpha}\right)$ and $C_{0 \mathrm{E}}\left(\mathcal{N}_{\alpha}\right)$.

Theorem 3 For the channel $\mathcal{N}_{\alpha}(0<\alpha \leq \pi / 4)$, the quantum Lovász number is strictly larger than the entanglement-assisted zero-error capacity (or even with no-signalling assistance), i.e.,

$$
\log \widetilde{\vartheta}\left(\mathcal{N}_{\alpha}\right)>C_{0, \mathrm{NS}}\left(\mathcal{N}_{\alpha}\right) \geq C_{0 \mathrm{E}}\left(\mathcal{N}_{\alpha}\right) .
$$

Proof It is easy to see this result from Proposition 1 and Proposition 2. To be specific, we have

$$
\begin{aligned}
\log \widetilde{\vartheta}\left(\mathcal{N}_{\alpha}\right) & =\log \left(2+\cos ^{2} \alpha+\cos ^{-2} \alpha\right) \\
& >2=C_{0, \mathrm{NS}}\left(\mathcal{N}_{\alpha}\right) \\
& \geq C_{0 \mathrm{E}}\left(\mathcal{N}_{\alpha}\right) .
\end{aligned}
$$

\section{GAP BETWEEN QUANTUM FRACTIONAL PACKING NUMBER AND FEEDBACK-ASSISTED OR NS-ASSISTED ZERO-ERROR CAPACITY}

A classical channel $\mathcal{N}=(X, p(y \mid x), Y)$ naturally induces a bipartite graph $\Gamma(\mathcal{N})=(X, E, Y)$, where $X$ and $Y$ are the input and output alphabets, respectively. And $E \subset X \times Y$ is the set of edges such that $(x, y) \in E$ if and only if the probability $p(y \mid x)$ is positive. The non-commutative bipartite graph in this case is given by

$$
K=\operatorname{span}\{|y\rangle\langle x|:(x, y) \text { is an edge in } \Gamma\} .
$$

Shannon first introduced the feedback-assisted zero-error capacity [2]. To be precise, his model has noiseless instantaneous feedback of the channel output back to the sender, and it requires some arbitrarily small rate of forward noiseless communication. For any classical channel with a positive zero-error capacity, he showed that the feedback-assisted zero-error capacity $C_{0 \mathrm{~F}}$ of a classical channel $\mathcal{N}$ is given by the fractional packing number of its bipartite graph [2]:

$$
\alpha^{*}(\Gamma)=\max \sum_{x} v_{x} \text { s.t. } \sum_{x} v_{x} p(y \mid x) \leq 1 \forall y, 0 \leq v_{x} \leq 1 \forall x .
$$

For any classical bipartite graph, the fractional packing number also gives the NS-assisted zeroerror classical capacity and simulation cost [16], i.e.,

$$
C_{0, \mathrm{NS}}(K)=S_{0, \mathrm{NS}}(K)=\log \alpha^{*}(\Gamma) .
$$


The quantum generalization of fractional packing number in [17] was suggested by Harrow as

$$
\begin{aligned}
\mathrm{A}(K) & =\max \operatorname{Tr} R_{A} \text { s.t. } 0 \leq R_{A}, \operatorname{Tr}_{A} P_{A B}\left(R_{A} \otimes \mathbb{1}_{B}\right) \leq \mathbb{1}_{B}, \\
& =\min \operatorname{Tr} T_{B} \text { s.t. } 0 \leq T_{B}, \operatorname{Tr}_{B} P_{A B}\left(\mathbb{1}_{A} \otimes T_{B}\right) \geq \mathbb{1}_{A} .
\end{aligned}
$$

This quantum fractional packing number $\mathrm{A}(K)$ has nice mathematical properties such as additivity under tensor product [17].

For any bipartite graph $\Gamma$, quantum fractional packing number also reduces to the fractional packing number, i.e.,

$$
\mathrm{A}(K)=\alpha^{*}(\Gamma)
$$

Furthermore, for a classical-quantum channel with non-commutative bipartite graph $K$, it also holds that [17]

$$
C_{0, \mathrm{NS}}(K)=\log \mathrm{A}(K) .
$$

However, if we consider general quantum channels, this quantum fractional packing number will exceed the NS-assisted zero-error capacity as well as the feedback-assisted zero-error capacity. An example is the class of channels $\mathcal{N}_{\alpha}$ and the proof is in the following Proposition 5. For $\mathcal{N}_{\alpha}$, it is easy to see that the set of linear operators $\left\{E_{i}^{\dagger} E_{j}\right\}$ is linearly independent, which means that $\mathcal{N}_{\alpha}$ is an extremal channel [53]. Thus, its non-commutative bipartite graph $K_{\alpha}$ is an extremal graph [17], which means that there can only be a unique channel $\mathcal{N}$ such that $K(\mathcal{N})=K_{\alpha}$.

For a general quantum channel, its feedback-assisted zero-error capacity depends only on its non-commutative bipartite graph. And the feedback-assisted zero-error capacity is always smaller than or equal to the entanglement-assisted classical capacity [23], i.e.,

$$
C_{0 \mathrm{~F}}(K) \leq C_{\operatorname{minE}}(K),
$$

where $C_{\operatorname{minE}}(K)$ is defined by

$$
C_{\operatorname{minE}}(K):=\min \left\{C_{\mathrm{E}}(\mathcal{N}): K(\mathcal{N})<K\right\} .
$$

Considering the fact that $C_{0, \mathrm{NS}}(K) \leq C_{\operatorname{minE}}(K) \leq S_{0, \mathrm{NS}}(K)$ [23], it is easy to see that $C_{\operatorname{minE}}\left(K_{\alpha}\right)$ is exactly two bits from Proposition 1.

Lemma 4 For non-commutative bipartite graph $K_{\alpha}(0<\alpha \leq \pi / 4)$, the quantum fractional packing number is given by

$$
\mathrm{A}\left(K_{\alpha}\right)=2+\cos ^{2} \alpha+\cos ^{-2} \alpha .
$$

Proof Let us choose $R_{A}=\left(2-\sin ^{2} \alpha\right)|0\rangle\langle 0|+x| 1\rangle\langle 1|$, then

$$
\operatorname{Tr}_{A} P_{\alpha}\left(R_{A} \otimes \mathbb{1}_{B}\right)=\frac{x \sin ^{2} \alpha}{1+\sin ^{2} \alpha}|0\rangle\langle 0|+| 1\rangle\left\langle 1\left|+\frac{x \cos ^{2} \alpha}{1+\cos ^{2} \alpha}\right| 2\right\rangle\langle 2| .
$$

When $x=1+\cos ^{-2} \alpha$, it is clear that $\operatorname{Tr}_{A} P_{\alpha}\left(R_{A} \otimes \mathbb{1}_{B}\right) \leq \mathbb{1}_{B}$. Therefore, $R_{A}$ is a feasible solution to the primal SDP of $\mathrm{A}\left(\mathcal{N}_{\alpha}\right)$, which means that

$$
\mathrm{A}\left(\mathcal{N}_{\alpha}\right) \geq \operatorname{Tr} R_{A}=2+\cos ^{2} \alpha+\cos ^{-2} \alpha .
$$

Similarly, it is easy to check that $T_{B}=\left(2-\sin ^{2} \alpha\right)|1\rangle\left\langle 1\left|+\left(1+\cos ^{-2} \alpha\right)\right| 2\right\rangle\langle 2|$ is a feasible solution to the dual SDP of $\mathrm{A}\left(\mathcal{N}_{\alpha}\right)$. Therefore,

$$
\mathrm{A}\left(\mathcal{N}_{\alpha}\right) \leq \operatorname{Tr} T_{B}=2+\cos ^{2} \alpha+\cos ^{-2} \alpha .
$$

Hence, we have that $\mathrm{A}\left(\mathcal{N}_{\alpha}\right)=2+\cos ^{2} \alpha+\cos ^{-2} \alpha$.

Now, we are able to show the separation. 
Proposition 5 For non-commutative bipartite graph $K_{\alpha}(0<\alpha \leq \pi / 4)$, we have that

$$
\begin{aligned}
C_{0 \mathrm{~F}}\left(K_{\alpha}\right) & <\log \mathrm{A}\left(K_{\alpha}\right), \\
C_{0, \mathrm{NS}}\left(K_{\alpha}\right) & <\log \mathrm{A}\left(K_{\alpha}\right) .
\end{aligned}
$$

Proof For general non-commutative bipartite graph $K$, it holds that $C_{0 \mathrm{~F}}(K) \leq C_{\min \mathrm{E}}(K)$ [23]. Then, by Proposition 1 and Lemma 4, we have

$$
C_{0 \mathrm{~F}}\left(K_{\alpha}\right) \leq C_{\operatorname{minE}}\left(K_{\alpha}\right)=2<\log \mathrm{A}\left(K_{\alpha}\right) .
$$

From Proposition 1 and Lemma 4, it is also clear that $C_{0, \mathrm{NS}}\left(K_{\alpha}\right)<\log \mathrm{A}\left(K_{\alpha}\right)$.

\section{DISCUSSIONS}

Interestingly, for the channel $\mathcal{N}_{\alpha}$, its quantum fractional packing number is equal to its quantum Lovász number. Let us recall that the Lovász number of a classical graph $G$ has an operational interpretation [17] as

$$
\vartheta(G)=\min \left\{\mathrm{A}(K): K^{\dagger} K<S_{G}\right\},
$$

where the minimization is over classical-quantum graphs $K$ and $S_{G}$ is non-commutative graph associated with $G$. A natural and interesting question is that for the non-commutative graph $S$, do we have

$$
\widetilde{\vartheta}(S)=\min \left\{\mathrm{A}(K): K^{\dagger} K<S\right\} ?
$$

The non-commutative bipartite graph of $\mathcal{N}_{\alpha}$ might be such an interesting example since Proposition 2 and Lemma 4 imply that $\widetilde{\vartheta}\left(\mathcal{N}_{\alpha}\right)=\mathrm{A}\left(K_{\alpha}\right)$.

It remains unknown whether the Lovász number coincides with $C_{0 \mathrm{E}}$ for every classical channel. For any confusability graph $G$, a variant of Lovász number called Schrijver number [54, 55] was proved to be a tighter upper bound on the entanglement-assisted independence number than the Lovász number [32] . However, it remains unknown whether Schrijver number will converge to the Lovász number in the asymptotic limit. Note that a gap between the Lovász number and the regularized Schrijver number would imply a separation between $C_{0 \mathrm{E}}(G)$ and $\vartheta(G)$. Moreover, it would be interesting to consider how to estimate the regularization of a sequence of semidefinite programs (or linear programs).

\section{CONCLUSIONS}

In summary, we have shown that there is a separation between the quantum Lovász number and the entanglement-assisted zero-error classical capacity. We have explicitly exhibited a class of quantum channels for which the quantum Lovász number is strictly larger than the entanglementassisted zero-error capacity. In particular, we have obtained the reversibility of these channels in the zero-error communication and simulation setting when assisted with quantum no-signalling correlations.

For any classical channel with a positive zero-error capacity, it is known that the feedbackassisted or NS-assisted zero-error capacity are both equal to the fractional packing number. In contrast, for quantum channels, we have shown that the feedback-assisted or the NS-assisted zero-error capacity is not given by the quantum fractional packing number in [17]. It also raises a new question to explore other quantum extensions of the fractional packing number. 


\section{Acknowledgment}

We would like to thank Andreas Winter for helpful suggestions. We also thank the referees of AQIS'16 for useful comments which improved the presentation of this paper. This work was partly supported by the Australian Research Council under Grant Nos. DP120103776 and FT120100449.

[1] C. E. Shannon, "A mathematical theory of communication," ACM SIGMOBILE Mob. Comput. Commun. Rev., vol. 5, no. 1, pp. 3-55, 1948.

[2] C. E. Shannon, "The zero-error capacity of a noisy channel," IRE Trans. Inf. Theory, vol. 2, no. 3, pp. 8-19, 1956.

[3] J. Körner and A. Orlitsky, "Zero-error information theory," IEEE Trans. Inf. Theory, vol. 44, no. 6, pp. 2207-2229, 1998.

[4] R. Duan and Y. Shi, "Entanglement between two uses of a noisy multipartite quantum channel enables perfect transmission of classical information," Phys. Rev. Lett., vol. 101, no. 2, p. 20501, 2008.

[5] R. Duan, "Super-activation of zero-error capacity of noisy quantum channels," arXiv preprint arXiv:0906.2527, 2009.

[6] T. S. Cubitt, J. Chen, and A. W. Harrow, "Superactivation of the asymptotic zero-error classical capacity of a quantum channel," IEEE Trans. Inf. Theory, vol. 57, no. 12, pp. 8114-8126, 2011.

[7] T. S. Cubitt and G. Smith, "An extreme form of superactivation for quantum zero-error capacities," IEEE Trans. Inf. Theory, vol. 58, no. 3, pp. 1953-1961, 2012.

[8] M. E. Shirokov and T. Shulman, "On superactivation of zero-error capacities and reversibility of a quantum channel," Commun. Math. Phys., vol. 335, no. 3, pp. 1159-1179, 2015.

[9] T. S. Cubitt, D. Leung, W. Matthews, and A. Winter, "Improving Zero-Error Classical Communication with Entanglement," Phys. Rev. Lett., vol. 104, no. 23, p. 230503, 2010.

[10] D. Leung, L. Mančinska, W. Matthews, M. Ozols, and A. Roy, “Entanglement can Increase Asymptotic Rates of Zero-Error Classical Communication over Classical Channels," Commun. Math. Phys., vol. 311, no. 1, pp. 97-111, 2012.

[11] R. Duan, S. Severini, and A. Winter, "Zero-error communication via quantum channels, noncommutative graphs, and a quantum Lovász number," IEEE Trans. Inf. Theory, vol. 59, no. 2, pp. 1164-1174, 2013.

[12] J. Briët, H. Buhrman, M. Laurent, T. Piovesan, and G. Scarpa, "Entanglement-assisted zero-error source-channel coding," IEEE Trans. Inf. Theory, vol. 61, no. 2, pp. 1124-1138, 2015.

[13] T. Piovesan, G. Scarpa, and C. Schaffner, "Multiparty Zero-Error Classical Channel Coding With Entanglement," IEEE Trans. Inf. Theory, vol. 61, no. 2, pp. 1113-1123, 2015.

[14] D. Stahlke, "Quantum zero-error source-channel coding and non-commutative graph theory," IEEE Trans. Inf. Theory, vol. 62, no. 1, pp. 554-577, 2016.

[15] W. Matthews, "A linear program for the finite blocklength converse of polyanskiy-poor-verd via nonsignaling codes," IEEE Trans. Inf. Theory, vol. 58, no. 12, pp. 70367044, 2012.

[16] T. S. Cubitt, D. Leung, W. Matthews, and A. Winter, "Zero-Error Channel Capacity and Simulation Assisted by Non-Local Correlations," IEEE Trans. Inf. Theory, vol. 57, no. 8, pp. 55095523, 2011.

[17] R. Duan and A. Winter, "No-Signalling-Assisted Zero-Error Capacity of Quantum Channels and an Information Theoretic Interpretation of the Lovász Number," IEEE Trans. Inf. Theory, vol. 62, no. 2, pp. 891-914, 2016.

[18] D. Leung and W. Matthews, "On the Power of PPT-Preserving and Non-Signalling Codes," IEEE Trans. Inf. Theory, vol. 61, no. 8, pp. 4486-4499, 2015.

[19] W. Xie, X. Wang, and R. Duan, "Converse bounds for classical communication over quantum networks," arXiv:1712.05637, Jun. 2017.

[20] X. Wang, W. Xie, and R. Duan, "Semidefinite programming strong converse bounds for classical capacity," IEEE Trans. Inf. Theory, vol. 64, no. 1, pp. 640-653, 2017.

[21] X. Wang, K. Fang, and R. Duan, "Semidefinite programming converse bounds for quantum communication," arXiv:1709.00200, Sep. 2017. 
[22] X. Wang, K. Fang, and M. Tomamichel, "On converse bounds for classical communication over quantum channels," arXiv:1709.05258, Sep. 2017.

[23] R. Duan, S. Severini, and A. Winter, "On zero-error communication via quantum channels in the presence of noiseless feedback," IEEE Trans. Inf. Theory, vol. 62, no. 9, pp. 5260-5277, 2016.

[24] S. Beigi and P. W. Shor, "On the complexity of computing zero-error and Holevo capacity of quantum channels," arXiv preprint arXiv:0709.2090, 2007.

[25] L. Lovász, "On the Shannon capacity of a graph," IEEE Trans. Inf. Theory, vol. 25, no. 1, pp. 1-7, 1979.

[26] L. Vandenberghe and S. Boyd, "Semidefinite programming," SIAM Rev., vol. 38, no. 1, pp. 49-95, 1996.

[27] S. Beigi, "Entanglement-assisted zero-error capacity is upper-bounded by the Lovász $\vartheta$ function," Phys. Rev. A, vol. 82, no. 1, p. 10303, 2010.

[28] D. Kretschmann and R. F. Werner, "Tema con variazioni: Quantum channel capacity," New J. Phys., vol. 6, 2004.

[29] C. H. Bennett, P. W. Shor, J. A. Smolin, and A. V Thapliyal, "Entanglement-assisted capacity of a quantum channel and the reverse Shannon theorem," IEEE Trans. Inf. Theory, vol. 48, no. 10, pp. 2637$2655,2002$.

[30] C. H. Bennett, I. Devetak, A. W. Harrow, P. W. Shor, and A. Winter, "The Quantum Reverse Shannon Theorem and Resource Tradeoffs for Simulating Quantum Channels," IEEE Trans. Inf. Theory, vol. 60, no. 5, pp. 2926-2959, 2014.

[31] M. Berta, M. Christandl, and R. Renner, "The quantum reverse Shannon theorem based on one-shot information theory," Commun. Math. Phys., vol. 306, no. 3, pp. 579-615, 2011.

[32] T. Cubitt, L. Mančinska, D. E. Roberson, S. Severini, D. Stahlke, and A. Winter, "Bounds on Entanglement-Assisted Source-Channel Coding via the Lovász Number and Its Variants," IEEE Trans. Inf. Theory, vol. 60, no. 11, pp. 7330-7344, 2014.

[33] L. Mančinska, G. Scarpa, and S. Severini, "New Separations in Zero-Error Channel Capacity Through Projective Kochen-Specker Sets and Quantum Coloring," IEEE Trans. Inf. Theory, vol. 59, no. 6, pp. 4025-4032, 2013.

[34] N. Alon, "The Shannon capacity of a union," Combinatorica, vol. 18, no. 3, pp. 301-310, 1998.

[35] H. Barnum, M. A. Nielsen, and B. Schumacher, "Information transmission through a noisy quantum channel," Phys. Rev. A, vol. 57, no. 6, p. 4153, 1998.

[36] L. G. Khachiyan, "Polynomial algorithms in linear programming," USSR Comput. Math. Math. Phys., vol. 20, no. 1, pp. 53-72, 1980.

[37] E. M. Rains, "A semidefinite program for distillable entanglement," IEEE Trans. Inf. Theory, vol. 47, no. 7, pp. 29212933, 2001.

[38] X. Wang and R. Duan, "A semidefinite programming upper bound of quantum capacity," in 2016 IEEE International Symposium on Information Theory (ISIT), vol. 2016-August, pp. 16901694.

[39] H. Barnum, M. Saks, and M. Szegedy, "Quantum query complexity and semi-definite programming," in 18th IEEE Annual Conference on Computational Complexity, 2003. Proceedings., 2003, no. August, pp. 179-193.

[40] X. Wang and R. Duan, "Irreversibility of Asymptotic Entanglement Manipulation Under Quantum Operations Completely Preserving Positivity of Partial Transpose," Phys. Rev. Lett., vol. 119, p. 180506, 2017.

[41] Y. C. Eldar, "A semidefinite programming approach to optimal unambiguous discrimination of quantum states," IEEE Trans. Inf. Theory, vol. 49, no. 2, pp. 446-456, 2003.

[42] Y. Li, X. Wang, and R. Duan, "Indistinguishability of bipartite states by positive-partial-transpose operations in the many-copy scenario," Phys. Rev. A, vol. 95, no. 5, p. 052346, May 2017.

[43] J. Watrous, "Simpler semidefinite programs for completely bounded norms," Chicago J. Theor. Comput. Sci., vol. 19, no. 1, pp. 1-19, 2013.

[44] O. Regev and T. Vidick, "Quantum XOR Games," in 2013 IEEE Conference on Computational Complexity, 2013, no. 844626, pp. 144-155.

[45] J. Watrous, Theory of quantum information. University of Waterloo, 2011.

[46] D. Beckman, D. Gottesman, M. A. Nielsen, and J. Preskill, "Causal and localizable quantum operations," Phys. Rev. A, vol. 64, no. 5, p. 52309, 2001.

[47] T. Eggeling, D. Schlingemann, and R. F. Werner, "Semicausal operations are semilocalizable," Europhys. Lett., vol. 57, no. 6, p. 782, 2002.

[48] M. Piani, M. Horodecki, P. Horodecki, and R. Horodecki, "Properties of quantum nonsignaling 
boxes," Phys. Rev. A, vol. 74, no. 1, p. 12305, 2006.

[49] O. Oreshkov, F. Costa, and C. Brukner, "Quantum correlations with no causal order," Nat. Commun., vol. 3, p. 1092, 2012.

[50] R. Duan and X. Wang, "Activated zero-error classical communication over quantum channels assisted with quantum no-signalling correlations," arXiv preprint arXiv:1510.05437, 2015.

[51] R. König, R. Renner, and C. Schaffner, "The operational meaning of min-and max-entropy," IEEE Trans. Inf. Theory, vol. 55, no. 9, pp. 4337-4347, 2009.

[52] M. Tomamichel, "A framework for non-asymptotic quantum information theory," arXiv preprint arXiv:1203.2142, 2012.

[53] M.-D. Choi, "Completely positive linear maps on complex matrices," Linear Algebra Appl., vol. 10, no. 3, pp. 285-290, 1975.

[54] A. Schrijver, "A comparison of the Delsarte and Lovász bounds," IEEE Trans. Inf. Theory, vol. 25, no. 4, pp. 425-429, 1979.

[55] R. J. McEliece, E. R. Rodemich, and H. C. Rumsey Jr, "The Lovász bound and some generalizations," J. Comb. Inform. Syst. Sci, vol. 3, no. 3, pp. 134-152, 1978. 